head bobbing and speech delay; epilepsy occurred in 8 (20\%), and developmental delay in $6(15 \%)$, skull deformity in $9(22.5 \%)$, and focal neurological signs in $8(20 \%)$. Indications for surgery included focal neurological deficits, skull deformities, and/or symptoms or signs of increased intracranial pressure. Epilepsy was an indication only if refractory to medication, if associated with radiological evidence of mass effect and/or increase in size of cyst. Complete resolution of signs and/or symptoms was obtained in $25(62.5 \%)$, and significant improvement in $12(30 \%)$. Headache was relieved in 10 $(66 \%)$ and improved in $4(26 \%)$, skull deformity improved in all 9 cases $(100 \%)$, and epilepsy control was improved in 7 (87\%). Postoperative complications included subdural hygroma in $5(12.5 \%)$, subdural hemorrhage in $4(10 \%)$ at 3 to 60 months after surgery, and failure of surgery in $4(10 \%)$. Endoscopic fenestration was as effective and safe but less invasive than cyst shunting. (Spacca B, Kandasamy J, Mallucci CL, Genitori L. Endoscopic treatment of middle fossa arachnoid cysts: a series of 40 patients treated endoscopically in two centres. Childs Nerv Syst Feb 2010;26:163-172). (Respond: Dr CL Mallucci, Department of Paediatric Neurosurgery, Royal Liverpool Children's Hospital “Alder Hey", Liverpool, UK. E-mail. mallucci@ntlworld.com).

COMMENT. A critical analysis of the surgical treatment of arachnoid cysts is provided in a commentary by Dr C Di Rocco, Rome, Italy (Childs Nerv System 2010;26:172-175). When analyzed objectively, the cause-effect relationship of the "classical" clinical manifestations of temporal arachnoid cyst is questionable. Headaches, reported in about $70 \%$ symptomatic cases, are almost always nonspecific and unrelated to cyst volume and intracystic pressure. In patients with epilepsy, the concordance between cyst location and semiology of seizures is rare whereas contralateral EEG abnormalities are common. Intracranial bleeding occurs in $2.2 \%$ of patients with MFAC, and this risk was not prevented by surgical operation in the present series. The rate of postoperative subdural hygroma requiring shunt or other surgical treatment is at least 2 -fold that reported for spontaneous or posttraumatic hygromas. Other neurosurgeons have expressed similar reservations regarding surgery and especially prophylactic surgery for temporal arachnoid cyst.

\title{
PRIMARY CARE UK RECORDS OF BRAIN TUMOR SIGNS AND SYMPTOMS
}

Symptoms and signs of brain tumor in children with and without brain tumors were compared in a UK population-based retrospective analysis of primary care records by researchers at University of York and other centers in the UK. Participants were 195 children, mean age 7.31 (range 1-14 years), newly diagnosed with brain tumors and 285 controls. Between birth and diagnosis with brain tumor symptoms and signs, cases with tumor consulted more often than controls, and their consultation rate with $>1$ suggestive symptom increased in the 2 years before diagnosis Symptom prevalence was higher among cases than controls, with 3.29 times as many consultations with $>1$ suggestive symptom. Suggestive symptoms in case children in order of prevalence included vomiting (74\% cases), headache (59\%), visual problems (44\%), unsteadiness $(42 \%)$, and anorexia $(31 \%)$. In controls, suggestive symptoms were vomiting (43\%), anorexia (18\%), headache $(15 \%)$, and visual $(15 \%)$ and hearing $(11 \%)$ problems. In 4 years before 
diagnosis, cases had at least twice as many consultations with $>1$ suggestive symptom in each 6 month period, and 20.81 times as many in the 6 months before diagnosis. Cases with combination of symptoms had an increased probability of tumor. Symptoms of brain tumor cases not observed in control children included head tilt, odd head movements, odd posture, back or neck stiffness, and unsteadiness. Recognition of unusual symptoms or specific symptom patterns and combinations is the key to identifying the child who needs prompt investigation. (Ansell P, Johnston T, Simpson J, Crouch S, Romaan E, Picton S. Brain tumor signs and symptoms: analysis of primary health care records from the UKCCS. Pediatrics Jan 2010;125(1):112-119). (Respond: Pat Ansell PhD, University of York, Dept of Health Sciences, York YO10 5DD, UK. E-mail: pat.ansell@egu.york.ac.uk).

COMMENT. Brain tumors in children are rare (2.4 per 100,000) and the average pediatrician or family practitioner will see few such cases in a lifetime of practice. With the advent of improved diagnostic methods and therapy, early diagnosis and prompt referral offer the potential for cure and a good quality of life. Knowledge of presenting symptoms of brain tumor should alert the primary care physician to suspect the diagnosis and refer the patient for neurological evaluation. (Cohen ME, Duffner PK. Brain Tumors in Children. Principles of Diagnosis and Treatment. New York; Raven Press, 1984;378). The account of the UK primary care experience emphasizes the early symptoms and signs suggestive of brain tumor and their increased prevalence and need for consultation with time. The importance of symptom combinations, unusual symptoms or specific symptom patterns is also stressed as the key to diagnosis. Early symptoms of brain tumor not addressed in the UK study include changes in affect, energy, motivation, or behavior. Indifference to playmates, listlessness, and somnolence should alert suspicion. The clinical course of a child with cerebellar medulloblsatoma, as portrayed by Cushing is worth review (Cohen and Duffner, 1984). Initial manifestations are nonlocalizing (changes in school work, vomiting, headache, enlarged head, and diplopia). These are followed by signs of a midline cerebellar syndrome, and terminally, tonsillar herniation, cerebellar fits, and decerebrate rigidity.

Seizures occurred in $50(17 \%)$ of 291 children with intracranial tumor treated at the Mayo Clinic from 1950 through 1959 (Millichap JG, Bickford RG, Miller RH, Backus RE. The EEG in children with intracranial tumors and seizures. Neurology $1962 ; 12: 329-336)$. EEG was of localizing value in $75 \%$ of supratentorial tumors and in $88 \%$ of those involving cerebral cortex. Seizures were not included in the list of symptoms observed in the UK primary care study.

\section{SUBDURAL EFFUSION AND INFANTILE NEURONAL CEROID LIPOFUSCINOSIS}

During the course of an ongoing NIH clinical study evaluating the benefit of cysteamine and $\mathrm{N}$-acetylcysteine in 9 patients with infantile neuronal ceroid lipofuscinosis (INCL), 4 were found to have subdural fluid collections without mass effect. Surgical drainage of fluid revealed evidence of old hemorrhage. Shearing of cortical veins as cerebral atrophy progresses is the mechanism suggested. No trauma or change in symptoms was associated with this finding. At follow-up 2.5 to 7 years later, 